\title{
Model Calculation of Cross Rolling Textures
}

\author{
H. Klein, C.Esling, H.J. Bunge \\ Department of Physical Metallurgy, TU Clausthal \\ Laboratoire de Métallurgie des Materiaux Poly- \\ cristallins, Université de Metz
}

\begin{abstract}
Model calculations of texture formation during cross-rolling are carried out based on the full-constrained Taylor theory. Three variants of cross-rolling were considered i.e. two-stage, four-stage and multistage rolling. Three types of glide systems $\{110\}\langle 111\rangle,\{112\}\langle 111\rangle$, $\{123\}\langle 111\rangle$ and all possible combinations of them were compared. The calculations were based on calculated orientation changes for grid points throughout the orientation space, taking the spread range according to the non-unique solution of the Taylor theory into account. The calculation were carried out in terms of the texture coefficients $C_{l}^{\mu \nu}$. The best agreement between experimental and calculated orientation densities was obtained with $\{123\}\langle 111\rangle$ glide systems.
\end{abstract}

\section{Introduction}

Plastic deformation of crystalline materials is based on glide and twinning. Both these processes give rise to orientation changes of the crystallites. This is the origin of the development of deformation texture. In the case of twinning, the orientation changes are discontinuous whereas glide deformation leads to continuous orientation changes. In the present paper only this latter case will be considered.

\section{The used mathematical model}

Model calculations of the orientation changes during plastic deformation have been based on different assumptions. Wheras the strainrate sensitive model has a unique solution for the amount of shear in 
each glide system and hence for the orientation change, the Taylor model is degenerated. In this latter case a crystal of a given orientation may assume a whole range of orientation changes, virtually with the same deformation energy. Hence, the orientation changes of any given orientation $g$ must be described by a multivalued orientation flow field in the orientation space, Fig. 1a.

$$
\delta g^{n} / d \eta=V^{n}(g) \quad \delta g^{n}=\left(\delta \varphi_{1}, \delta \phi, \delta \varphi_{2}\right)
$$

The index $n$ numerates different energetically equivalent solutions. Hence, any linear combination of flow vectors $V^{n}(g)$ may be chosen as a possible orientation change. These orientation changes fill a whole spread range $\sigma$

If we start with a given orientation distribution in the orientation space and apply to it the flow field with a small deformation step $d \eta$ then we end up with a slightly modified orientation distribution. This can be described most concisely in terms of the texture coefficients $C_{l}^{\mu \nu}[2,3,4]$

$$
\frac{d C_{l}^{\mu \nu}}{d \eta}=\sum_{\lambda=0}^{\lambda_{\max }} \sum_{\sigma=1}^{M(l)} \sum_{\rho=1}^{N(l)} a_{l \lambda}^{\sigma \rho \mu \nu} C_{\lambda}^{\sigma \rho}(\eta)
$$

with

$$
f(g, \eta)=\sum_{l=0}^{L_{\text {max }}} \sum_{\mu=1}^{M(l)} \sum_{\nu=1}^{N(l)} C_{l}^{\mu \nu}(\eta) \dot{T}_{l}^{\mu \nu}(g)
$$

In order to calculate the matrix $[a]$ in eq. 2 , we start with a texture consisting of only one orientation $g$ Fig. 1b. In this case the coefficients $C_{l}^{\mu \nu}$ in eq. 3 have the form [1]

$$
C_{l}^{\mu \nu}=(2 l+1) \dot{T}_{l}^{\mu \nu}(g)
$$

The modified texture is a certain distribution within the spread range $\sigma$, the shape of which has to be fixed by additional assumptions. In a first approximation we assume that it has the shape of a Gaussdistribution with spread $\omega$ about a mean orientation $g^{\prime}$. The coefficients of such a texture can be written in the form

$$
C_{l}^{\mu \nu}=e(l, \omega) \ddot{T}_{l}^{\mu \nu}\left(g^{\prime}\right)
$$

with

$$
e(l, \omega)=\frac{\exp \left(-1 / 4 l^{2} \omega^{2}\right)-\exp \left(-1 / 4(l+1)^{2} \omega^{2}\right)}{1-\exp \left(-1 / 4 \omega^{2}\right)}
$$


where $\omega$ and $g^{\prime}$ are functions of the starting orientation $g$. With these assumptions eq. 2 takes on the form

$e(l, \omega) \ddot{T}_{l}^{\mu \nu}\left(g^{\prime}\right)-(2 l+1) \dot{T}_{l}^{\mu \nu}(g)=d \eta \sum_{\lambda=0}^{\lambda_{\max }} \sum_{\sigma=1}^{M(l)} \sum_{\rho=1}^{N(l)} a_{l \lambda}^{\sigma \rho \mu \nu}(2 \lambda+1) \dot{T}_{\lambda}^{\sigma \rho}(g)$

Then the starting orientation $g$ is chosen in equidistant steps $\Delta \varphi_{1}$, $\Delta \phi, \Delta \varphi_{2}$ throughout the whole orientation space and each time $g^{\prime}$ and $\omega$ are calculated with the assumed model (e.g. the Taylor theory). Eq. 7 can then be solved for the unknown coefficients $a_{l \lambda}^{o \rho \mu \nu}$.

If the coefficients $a_{l \lambda}^{\sigma \rho \mu \nu}$ are known, eq.2 can be integrated numerically by adding successively small deformation steps $\Delta \eta$.

The flow field $V^{n}(g)$ and hence the matrix $[a]$ in eq. 2 depend on the crystallographic nature of the assumed glide system and on the deformation tensor. In the present calculations, plane strain deformation was assumed which is an approximation to rolling deformation. This kind of deformation leads to a texture which has orthorhombic sample symmetry. The same symmetry is also maintained if the rolling direction is turned by $90^{\circ}$ as is the case in cross-rolling. This can be achieved by keeping the rolling direction fixed and turning the sample and with it the texture through $90^{\circ}$. This can easily be expressed in term of the coefficients

$$
C_{l}^{\mu \nu}\left(90^{\circ}\right)=(-1)^{\nu-1} C_{l}^{\mu \nu}\left(0^{\circ}\right)
$$

With the matrix $[a]$ for plane strain deformation and the transformation eq. 8 any sequence of small rolling steps in longitudinal and transverse direction can easily be modelled. For comparison with experimental results $[5,6,7]$, three different sequences were chosen.

1. The rolling direction was changed after each deformation step (multi-stage or true cross-rolling)

2. The rolling direction was changed three times after equal deformation degrees (four-stage cross-rolling).

3. The rolling direction was changed only once, after half the total deformation degree (two-stage cross-rolling)

Hence, in all three cases the accumulated deformation in longitudinal and transverse direction was the same only the sequence of rolling steps was different. 


\section{Results and Conclusion}

Numerical calculations were carried out on the basis of the classical (full-constrained) Taylor theory assuming plane strain deformation and the glide systems

$\{110\}\langle 111\rangle,\{112\}\langle 111\rangle,\{123\}\langle 111\rangle$

as well as all possible combinations of these. The deformation step was chosen $\Delta \eta=0.05 \sim 5 \%$. In Fig. 2 one section of the multivalued flow field is shown corresponding to $\{110\}\langle 111\rangle$ glide. One sees clearly that in certain regions of the orientation space the flow field is nearly unique with only a very small spread range $\sigma$ whereas in higly symmetric orientations there is no average shift but a broad spread range $\sigma$.

In Fig.3 a,b the resulting textures are shown for multistage rolling with total rolling degrees of $70 \%$ and $90 \%$ respectively. The texture consists of an ideal orientation $\{001\}\langle 110\rangle$ and a fibre component $\langle 111\rangle \|$ ND. Hence, it is sufficient to show only three sections $\varphi_{1}=0^{\circ}, 45^{\circ}, 90^{\circ}$ of the texture. The results are qualitatively the same for all kinds of glide systems. However, the maximum orientation densities as well as the spread ranges are different for different glide systems The results are also in good agreement with experimental results obtained in ARMCO-iron [5,7]. It is seen that the calculated densities reach approximately the same magnitude as the experimental ones. This is due to the inclusion of the spread $\sigma$ into the calculations. Without this spread the calculated densities were found much too high . Comparing the values in Fig.4 and Fig.5 it is seen that the best agreement is obtained with the glide system $\{123\}\langle 111\rangle$.

Fig.6 shows the orientation distributions calculated for four-stage rolling. Fig.7 shows the densities in the three main orientations and Fig. 8 gives the corresponding experimental densities. Again the $\{123\}\langle 111\rangle$-results are nearest to the experimental ones although the densities of the fibre component $\langle 111\rangle \| N D$ are higher than the experimental ones.

Figs.9,10,11, finally give the calculated and measured values for two-stage rolling. Also in this case the qualitative agreement ist good although the quantitative deviations are larger.

Finally, Figs. 12,13 compare the calcuated densities of the orientation $\{001\}\langle 110\rangle$ for $\{123\}\langle 111\rangle$ glide with the experimental ones. The quantitative agreement is best for multi-stage rolling. In four- 
stage rolling the calculated values are slightly higher and in two-stage rolling they are smaller than the experimental ones.

Comparing the calculated results with the experimental ones one has to take into account that experimental rolling is not exactly plane-strain deformation. Furthermore, the full constrained Taylor theory is only an approximation and the assumption of Gaussdistribution in the spread range $\sigma$ is an additional simplification. Taking these premises into account, the agreement between experimental and calculated orientation densities seem to be quite satisfactory.

\section{References}

[1] H.J. Bunge, Texture Analysis in Material Science, Butterworth, London (1982)

[2] A. Clement, P. Coulomb, in: Texures of Materials, Vol.I, S.329, Ed.:G. Gottstein u. K. Lücke, Springer Verlag, Berlin (1978)

[3] H.J. Bunge, C.Esling, Scripta Met., 18, (1984), S.191

[4] A. Clement, P.Coulomb, Scripta Met., 13, (1979), S.899

[5] A. Böcker, H. Klein, H.J. Bunge, Textures and Microstructures, 12, (1990), S.103

[6] A. Böcker, H. Klein, H.J. Bunge, Textures and Microstructures, 12, (1990), S.155

[7] A. Böcker, H.J. Bunge, in: Proc. 9th Intern. Conf. on Textures of Materials, Avignon (1190) 


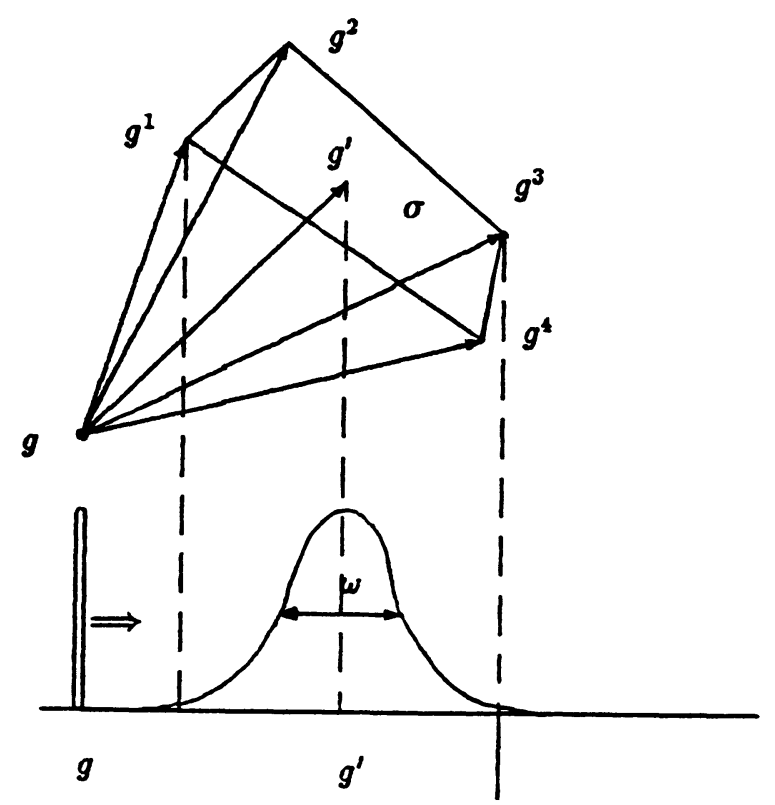

a)

b)

Figure 1:

a) Orientation change of a given orientation $g$ described by a multivalued orientation flow field

b) Approximation of Gauss-distribution in the spread rang $\sigma$

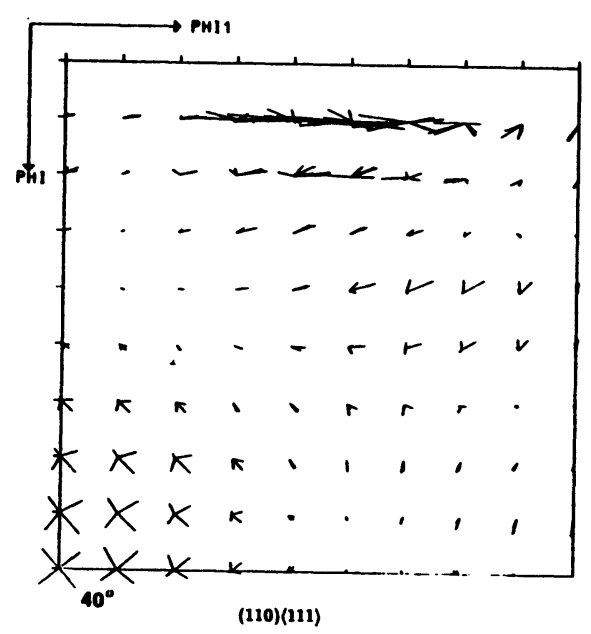

Figure 2:

Section of a multivalued flow field corresponding to $\{110\}\langle 111\rangle$ glide, $\varphi_{2}=40^{\circ}$. 
a)

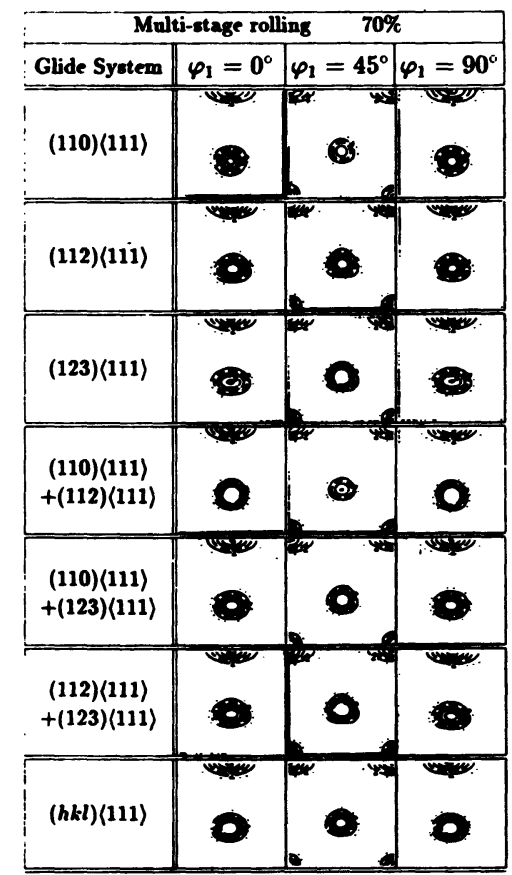

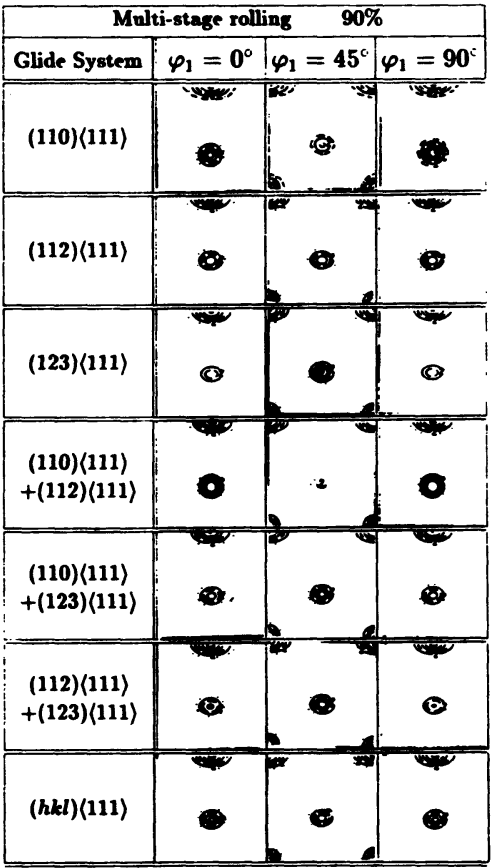

b)

\section{Figure 3:}

Model textures for multi-stage rolling and different glide systems a) total rolling degree $70 \%$

b) total rolling degree $90 \%$

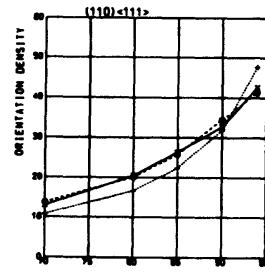

deronarition (x)

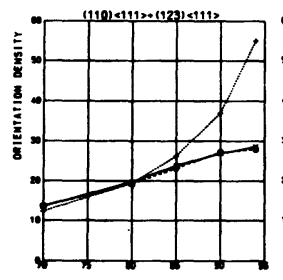

oeronenation (x)

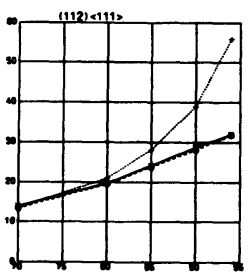

oeronation (x)

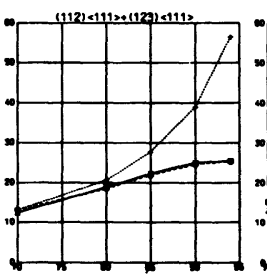

oeroenation (x)

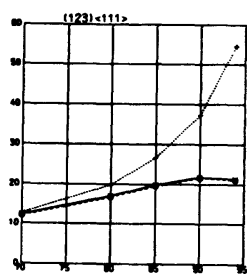

oef onnarion (x)

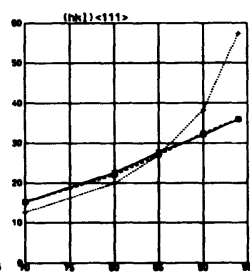

oeroenniriom ix

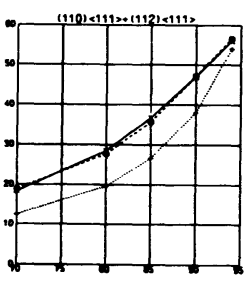

oefornation (x)

Multi-stage rolling calc.

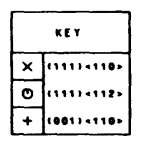

Figure 4: Model ralculation of multi-stage rolling: Orientation densities for the texture components $\{110\}\langle 111\rangle,\{111\}\langle 112\rangle$ and $\{001\}\langle 110\rangle$ as a function of the degree of the deformation for different glide systems. 


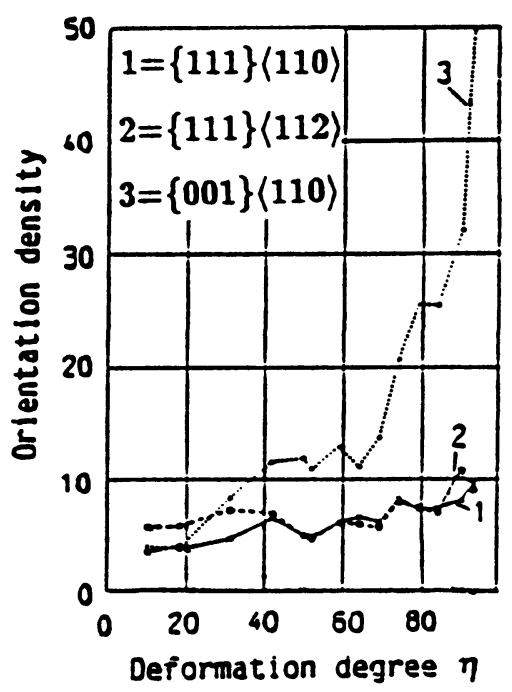

Figure 5:

Experimental multi-stage rolling: Orientation densities for the texture components $\{111\}\langle 110\rangle,\{111\}\langle 112\rangle$ and $\{001\}\langle 110\rangle$ as a function of the deformation degree.

a)

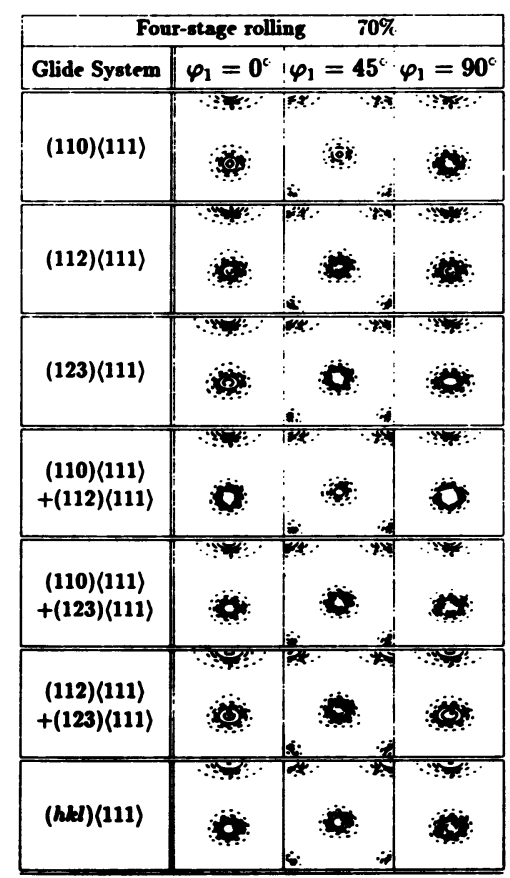

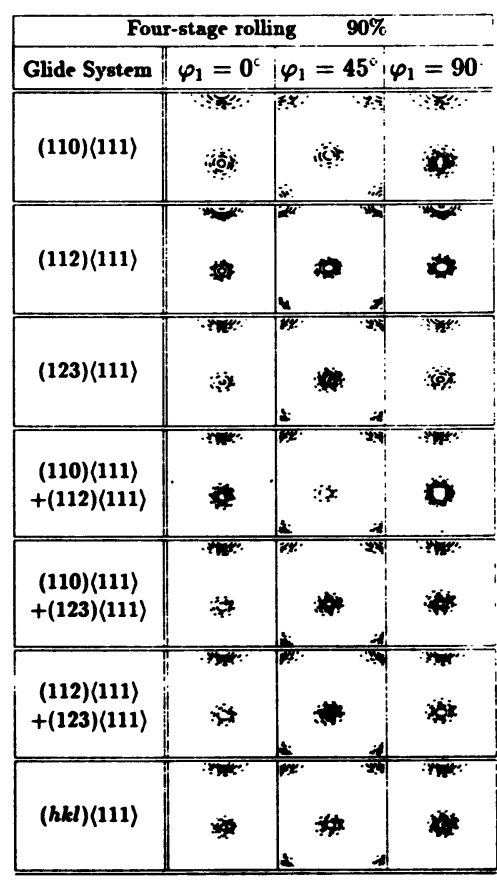

b)

Figure 6:

Model textures for four-stage rolling and different glide systems a) total rolling degree $70 \%$

b) total rolling degree $90 \%$ 


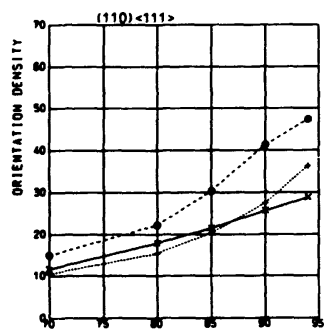

OEFormation (x)

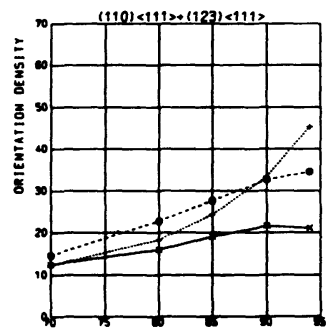

Deformation (x)

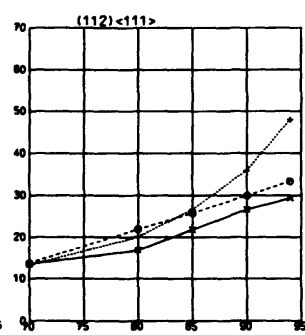

DEF ORMATION ( $x$ )

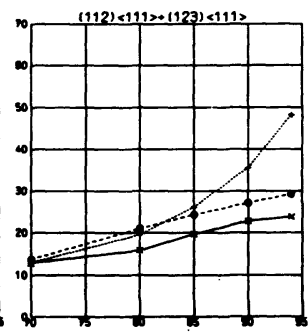

Der ORMATION (x)

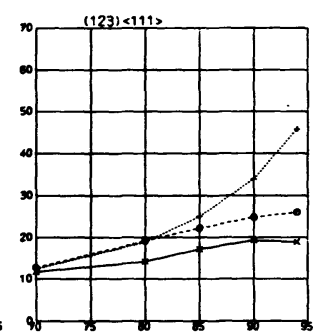

DEF ORMATION $(x)$

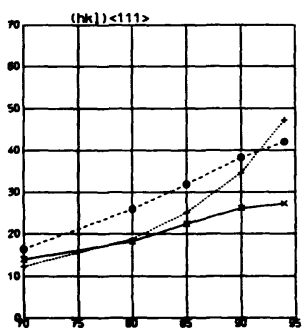

DEF ORMATION $\mid \mathbf{x}$

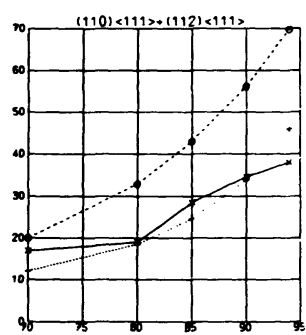

DEF ORMATION ( $x$ )

Four-stage rolling calc.

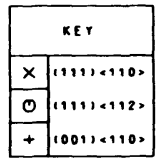

Figure 7:

Model calculation of four-stage rolling: Orientation densities for the texture components $\{110\}\langle 111\rangle,\{111\}\langle 112\rangle$ and $\{001\}\langle 110\rangle$ as a function of the degree of the deformation for different glide systems.

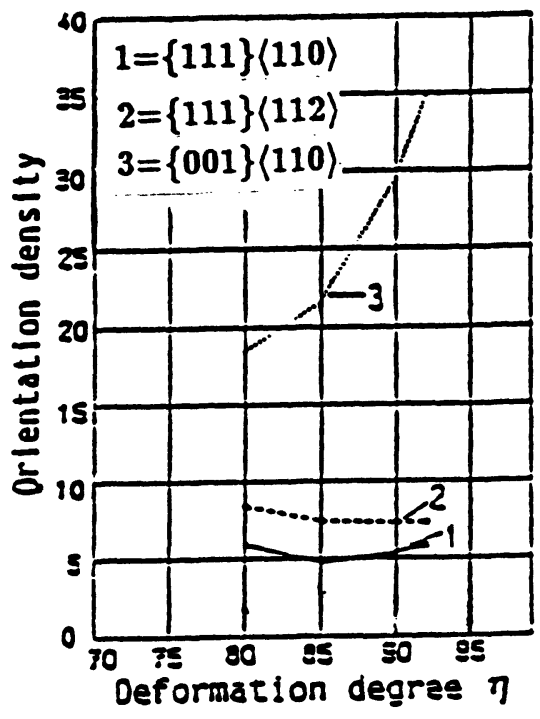

\section{Figure 8:}

Experimental four-stage rolling: Orientation densities for the texture components $\{111\}\langle 110\rangle,\{111\}\langle 112\rangle$ and $\{001\}\langle 110\rangle$ as a function of the deformation degree. 
a)

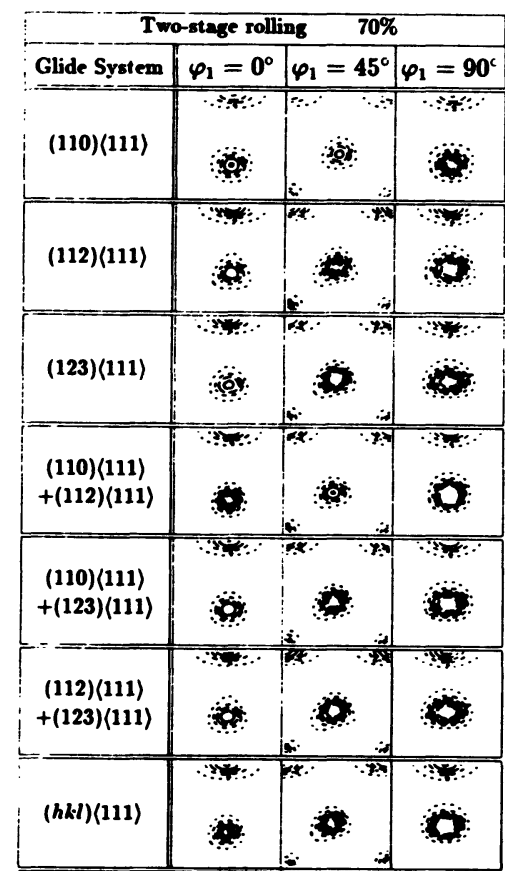

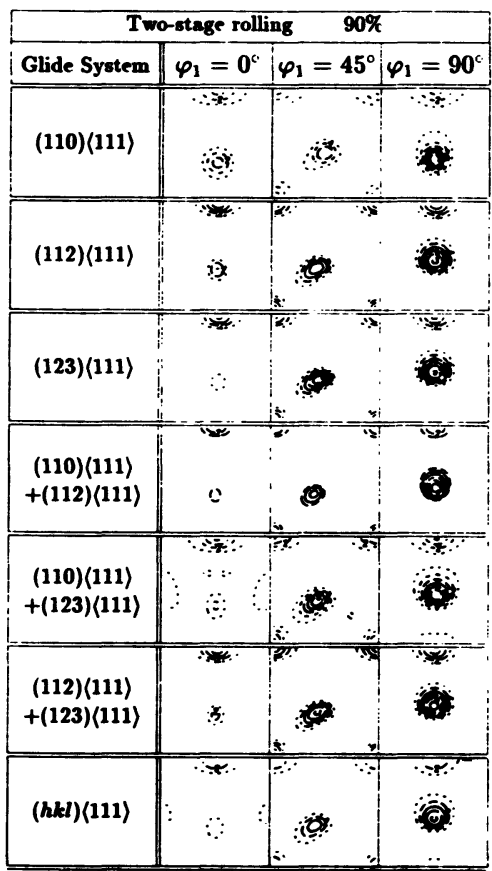

b)

\section{Figure 9:}

Model textures for two-stage rolling and different glide systems a) total rolling degree $70 \%$

b) total rolling degree $90 \%$
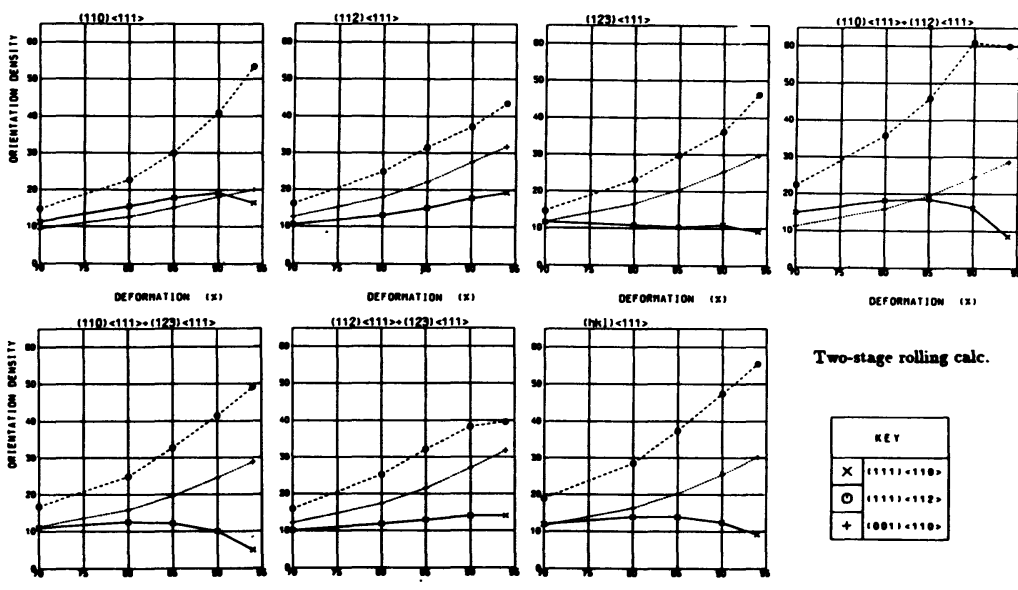

oeroonation (x)

oeformation (x)

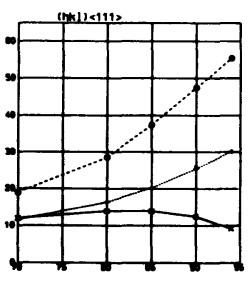

Two-stage rolling calc.

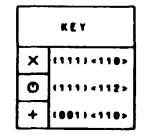

Figure 10: Model calculation of two-stage rolling: Orientation densities for the texture components $\{110\}\langle 111\rangle,\{111\}\langle 112\rangle$ and $\{001\}\langle 110\rangle$ as a function of the degree of the deformation for different glide systems. 


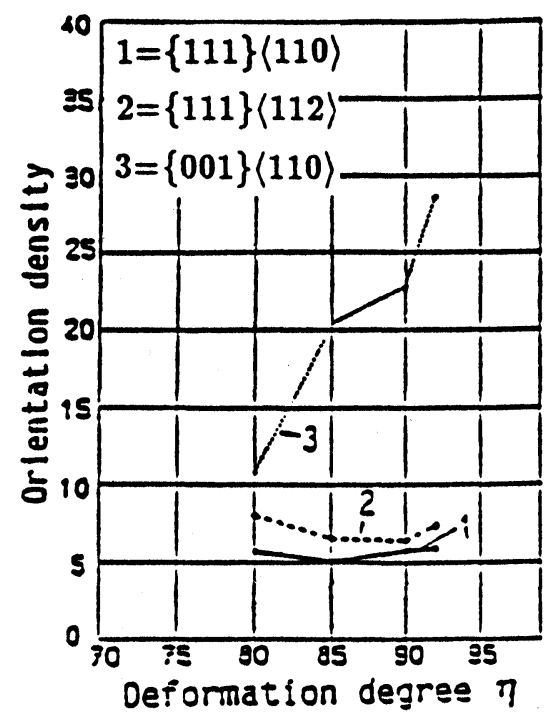

Figure 11:

Experimental two-stage rolling: Orientation densities for the texture components $\{111\}\langle 110\rangle,\{111\}\langle 112\rangle$ and $\{001\}\langle 110\rangle$ as a function of the deformation degree.

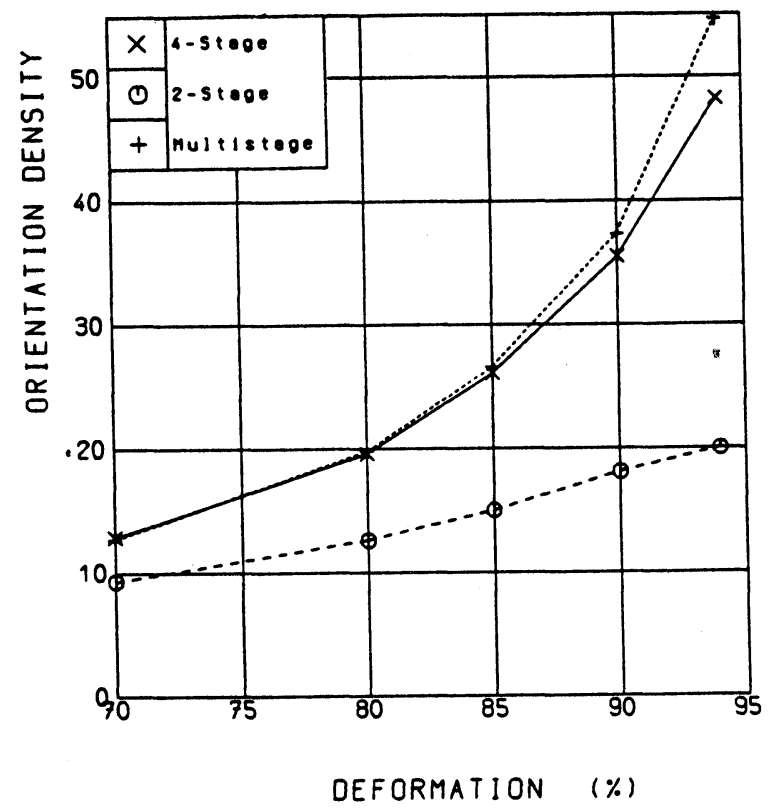

Figure 12:

DEFORMATION (\%)

Model calculation of the 3 cross rolling modes for $\{123\}\langle 111)$ glide. Orientation densities of the $\{001\}\langle 110\rangle$ texture component as a function of the degree of the deformation. 


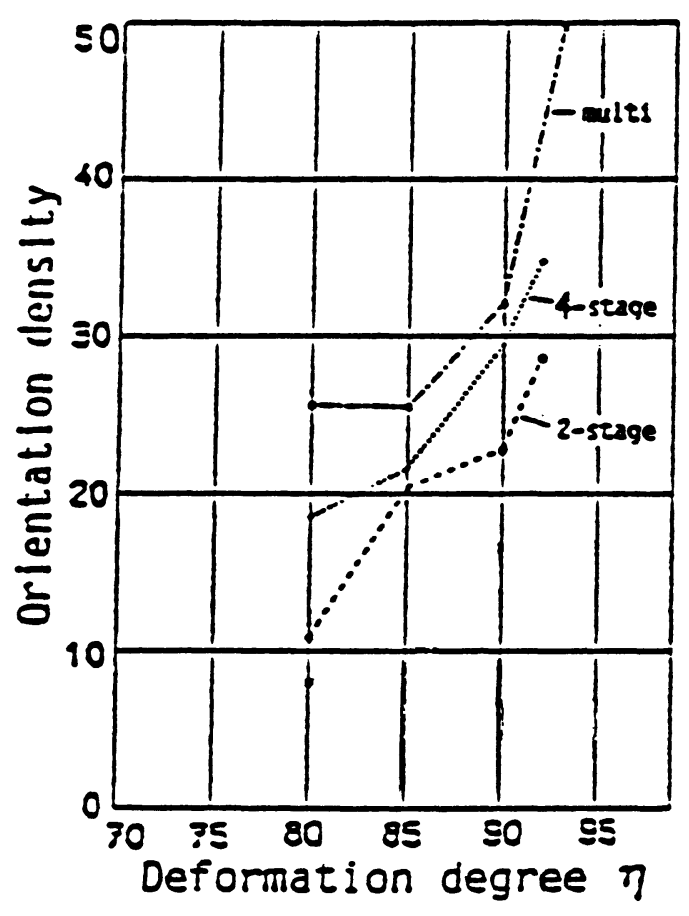

Figure 13:

Experimental cross rolling ( 3 modes). Orientation densities of the $\{001\}\langle 110\rangle$ texture component as a function of the degree of the deformation. 